\title{
Two Highly Stable Silver Nanoparticles: Surface Plasmon Resonance Spectra Study of Silver Nanoparticles Capped with m-Hydroxybenzoic Acid and p-Hydroxybenzoic Acid
}

\author{
Gusrizal Gusrizal $^{* 1}$, Sri Juari Santosa ${ }^{2}$, Eko Sri Kunarti², Bambang Rusdiarso ${ }^{2}$ \\ ${ }^{1}$ Department of Chemistry, Faculty of Mathematics and Natural Sciences, Universitas Tanjungpura, \\ Jl. Prof. Dr. H. Hadari Nawawi, Pontianak, Indonesia \\ ${ }^{2}$ Department of Chemistry, Faculty of Mathematics and Natural Sciences, Universitas Gadjah Mada, \\ SekipUtara, PO BOX BLS 21 Yogyakarta, Indonesia \\ *Corresponding author: e-mail: gusrizal@chemistry.untan.ac.id
}

Received March 18, 2018; Accepted May 18, 2018; Available online May 31, 2018

\begin{abstract}
Silver nanoparticles capped with m-hydroxybenzoic acid and p-hydroxybenzoic have been successfully synthesized, but the long-term stability data of these silver nanoparticles are not available. In this paper, we report the stability of these two types of silver nanoparticles for a period of 40 weeks observation based on the change of surface plasmon resonance spectra of silver nanoparticles. Silver nanoparticles were synthesized by reduction of silver nitrate with m-hydroxybenzoic acid and p-hydroxybenzoic acid without addition of capping agent. The presence of silver nanoparticles was indicated by the appearance of yellow color due to the surface plasmon resonance of silver nanoparticles. The resulted silver nanoparticles were stored at room temperature and further UV-visible spectrophotometer was used to follow the change in surface plasmon resonance spectra. The surface plasmon resonance spectra of silver nanoparticles were overlapped for the first 18 weeks, followed by little change in the position of absorption maxima $\left(\lambda_{\max }\right)$, peak intensity, and width of the absorption peak until the week of 40. Silver nanoparticles capped with mhydroxybenzoic acid and silver nanoparticles capped with p-hydroxybenzoic acid were highly stable which should make them suitable for further applications. The results show the potential of m-hydroxybenzoic acid and p-hydroxybenzoic acid to become a new reducing agent in the synthesis of highly stable silver nanoparticles. The m-hydroxybenzoic acid and p-hydroxybenzoic acid appeared to act as both reducing and capping agent.
\end{abstract}

Keywords: hydroxybenzoic acid, nanoparticle, silver, surface plasmon resonance

\section{INTRODUCTION}

Over the past few years, the scientific publication shows a meaningful increase of research in exploring nanoparticles. It is generally accepted that nanoparticles are particulate matter with dimension of 1-100 nm. The interest in nanoparticles is resulted from the unique properties at nanoscale that not found in their bulk material with the same chemical composition. These properties include high surface to volume ratio, high surface energy, unique mechanical, magnetic, electrical, thermal, and optical behavior (Chen, Roy, Yang, \& Prasad, 2016). Silver nanoparticles have received much attention among the metal nanoparticles. The characteristic features of silver nanoparticles are the presence of an intense and broad absorption peak in the visible range which called surface plasmon resonance spectra. The spectra stem from the interaction of light incident on the nanoparticles with the conduction electron of the metal and it depends on the size, shape, and media of the nanoparticles (Krutyakov, Kudrinskiy, Olenin, \& Lisichkin, 2008; Mock, Barbic, Smith, Schultz, \& Schultz, 2002).

Silver nanoparticles have been applied in the development of chemical analysis method as a sensor for colorimetric determination. The determination is based on the change of surface plasmon resonance spectra resulted from the aggregation of silver nanoparticles. The plasmonic properties of silver nanoparticles can be affected by the size of particles (Rycenga, Cobley, Zeng, Li, \& Moran, 2012). Interaction of silver nanoparticles with analytes induces the aggregation of silver nanoparticles and result in the decrease of surface plasmon resonance peak intensity. The change of peak intensity relates to the concentration of analyte (Sachdev et al., 2016; Shrivas, Sahu, Patra, Jaiswal, \& Shankar, 2016). In addition, silver nanoparticles also possess antibacterial properties which has inspired the application of silver nanoparticles in the field of food production, water treatment, textile industries, consumer goods and biomedical application as 
an antibacterial agent. The antibacterial properties of silver nanoparticles depend on their size and shape (Agnihotri, Mukherji, \& Mukherji, 2014; Marambio-Jones \& Hoek, 2010).

In the preparation of silver nanoparticles, the method of synthesis allow to control their size and shape (Sun \& Xia, 2002; Wiley et al., 2005). A large number of methods for the synthesis of silver nanoparticles are now available. A common approach used by chemist in synthesis of silver nanoparticles is chemical reduction. The precursor of silver, reducing agent, and stabilizer or capping agent should be available in the chemical reduction method. Silver ion is reduced by reducing agent and then stabilized with a capping agent to prevent the aggregation of resulted silver nanoparticles (Rycenga et al., 2012). Sodium borohydride, sodium citrate (Lee \& Meisel, 1982), ethylene glycol (Sun \& Xia, 2002), and aniline (Yang, Yin, Jia, \& Wei, 2011) have been used as reducing agent. Polyethylene glycol and polyvinylpyrrolidone have been widely used as capping agent (Tejamaya, Römer, Merrifield, \& Lead, 2012). In several cases, the synthesis of silver nanoparticles can be carried out without the addition of capping agent since reducing agent also plays a role as a capping agent simultaneously (Adegboyega et al., 2013; Annadhasan, Muthukumarasamyvel, Babu, \& Rajendiran, 2014). The choice of reducing and capping agent allows researchers to control the size and shape of silver nanoparticles. The different reducing and capping agent generate the silver nanoparticles with different size and shape (Bastus, Merkoçi, Piella, \& Puntes, 2014; Rycenga et al., 2012).

Although some publication of synthesis method of silver nanoparticles mentions the stability, the evaluation of the long-term period of stability is still limited. Pinto et al. (2010) studied the stability for 1 year period of silver nanoparticles produced by reduction silver ion with sodium borohydride in the presence of trisodium citrate. Yang et al. (2011) monitored the 1 year storage of silver nanoparticles synthesized using aniline and dodecylbenzene sulfonic acid as reducing and capping agent, respectively. Adegboyega et al. (2013) observed 210 days of the stability of silver nanoparticles synthesized using fulvic acids and Bastus et al. (2014) studied 7 weeks storage stability of silver nanoparticles synthesized using the combination of sodium citrate and tannic acid as reducing agent.

We have successfully synthesized silver nanoparticles capped with m-hydroxybenzoic acid and p-hydroxybenzoic acid by reduction of silver nitrate separately with mhydroxybenzoic acid and p-hydroxybenzoic acid without additional capping agent (Gusrizal, Santosa, Kunarti, \& Rusdiarso, 2016; Gusrizal, Santosa, Kunarti E, \& Rusdiarso, 2017). The rationale behind the selection of using m-hydroxybenzoic acid and p-hydroxybenzoic acid in the synthesis of silver nanoparticle without additional capping agent is the fact that they possess hydroxyl phenol and carboxylic group as reducing and capping group in one molecule. These two functional groups are able to be the reducing and capping sites in the synthesis of silver nanoparticles. The aim of this present study is determine the long-term stabilization effect of m-hydroxybenzoic acid and p-hydroxybenzoic acid as capping agent that simultaneously act as reducing agent in the synthesis of silver nanoparticles, which has not been previously reported. The stability of silver nanoparticles was determined by observing the intensity, the position of absorption maxima $(\lambda \max )$, and full-width half maximum (FWHM) absorption peak of surface plasmon resonance spectra.

\section{EXPERIMENTAL SECTION}

\section{Material and Instrumentation}

All chemicals in this work such as sodium hydroxide (Merck), m-hydroxybenzoic acid (Sigma-Aldrich), p-hydroxybenzoic acid (Sigma-Aldrich), and silver nitrate (Merck) were used as received and no further purification was performed. Double distilled water was used in all experiment. Surface plasmon resonance spectra of $\mathrm{s}$ were obtained by UV-visible spectrophotometric measurement (Shimadzu UV-PharmaSpec) using $1 \mathrm{~cm}$ optical path length cuvettes.

\section{Synthesis of Silver Nanoparticles}

Silver nanoparticles were synthesized by reduction of silver ion with $\mathrm{m}$-hydroxybenzoic acid and p-hydroxybenzoic acid, similar to the previously published work (Gusrizal et al., 2016, 2017). The synthesis was carried out using $2.5 \times 10^{-4} \mathrm{M}$ silver nitrate and two concentrations of each reducing agent e.g $1.0 \times 10^{-3}$ and $1.0 \times 10^{-2} \mathrm{M}$. The synthesis was initiated by an adjustment of the $\mathrm{pH}$ of 
reducing agent to 11 with the addition of sodium hydroxide solution. In a test tube, $5 \mathrm{~mL}$ of silver nitrate was mixed with $5 \mathrm{~mL}$ of the $\mathrm{pH}$-adjusted reducing agent. The mixture was warmed up in boiling water bath and then cooled with tap water. Warming up the mixture was conducted for 15 minutes and 1 hours if the reaction using m-hydroxybenzoic acid and p-hydroxybenzoic acid as a reducing agent respectively. The resulted silver nanoparticles were stored in closed bottles at room temperature. At a certain time period, surface plasmon resonance spectra of silver nanoparticles were measured at room temperature by UV-visible spectrophotometer. The spectra were recorded in the range of wavelength 300 to $750 \mathrm{~nm}$ with the resolution of $1 \mathrm{~nm}$.

\section{RESULTS AND DISCUSSION}

\section{Synthesis of Silver Nanoparticles}

In this work, silver nitrate was used as the precursor of silver. The synthesis of silver nanoparticles was carried out by mixing silver nitrate with m-hydroxybenzoic and phydroxybenzoic acid separately and then warming up it in boiling water bath. The mhydroxybenzoic and p-hydroxybenzoic acid were used as both a reducing and capping agent. Hydroxybenzoic acid is a bifunctional molecule which contains hydroxyl phenolic
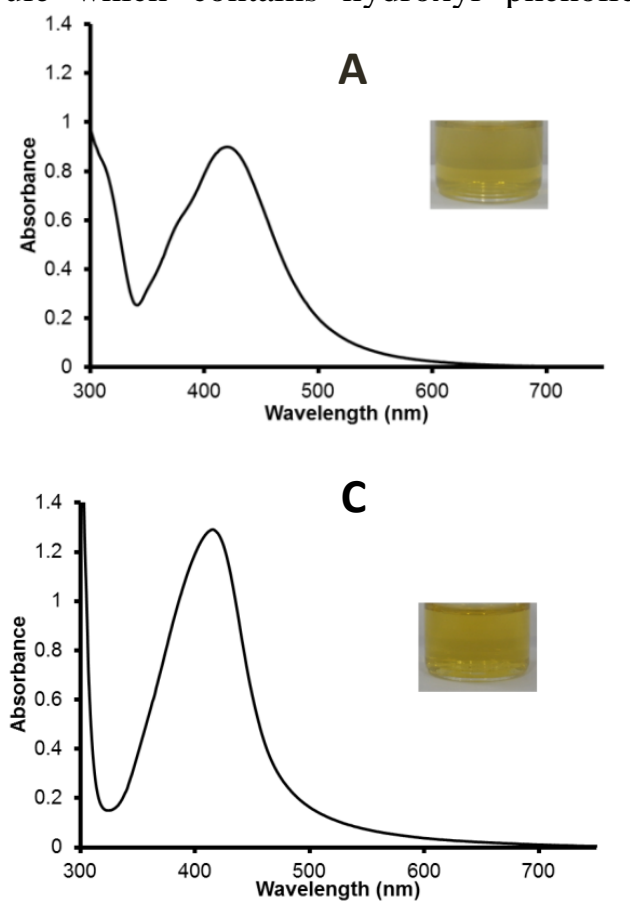

and carboxylic group. It is reasonable to expect that m-hydroxybenzoic acid and phydroxybenzoic acid are able to act simultaneously as capping agent in addition to reducing agent. Silver ion is reduced through hydroxyl phenolic group and resulted silver nanoparticles are then capped with hydroxybenzoic acid through its carboxylic group. Since m-hydroxybenzoic and phydroxybenzoic acid reduced silver ion, the color of the solution changed immediately from colorless to yellow which is considered to be the indication of the presence of silver nanoparticles. The presence of silver nanoparticles was further confirmed by the UV-visible measurement as shown in Figure 1. The appearance of yellow color and UVvisible spectra is a characteristic of silver nanoparticles which due to the surface plasmon resonance absorption (Krutyakov et al., 2008). The formation of silver nanoparticles was also proved by transmission electron microscopy (TEM) image as shown in our previous publication (Gusrizal et al., 2016, 2017). Calculation the average particles size from size distribution according to the TEM image resulted in the average size of $31 \pm 15$ and $32 \pm 12 \mathrm{~nm}$ for silver nanoparticles synthesized using m-hydroxybenzoic acid and phydroxybenzoic acid respectively.
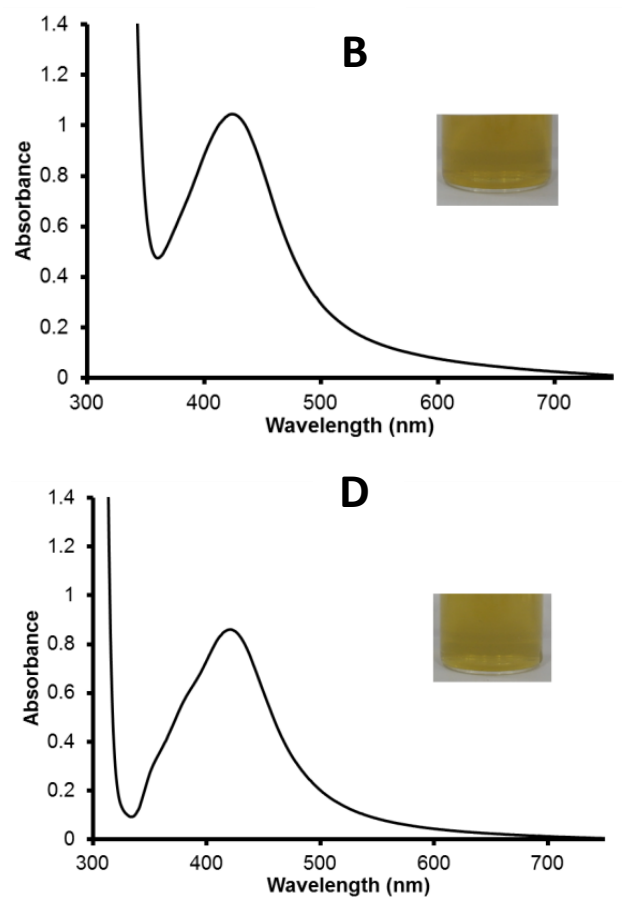

Figure 1. Surface plasmon resonance spectra of silver nanoparticles synthesized with different reducing agent. (A) m-hydroxybenzoic acid $1.0 \times 10^{-3} \mathrm{M} ;(\mathbf{B}) \mathrm{m}$-hydroxybenzoic acid $1.0 \times 10^{-2} \mathrm{M}$; (C) p-hydroxybenzoic acid $1.0 \times 10^{-3} \mathrm{M}$; and (D) p-hydroxybenzoic acid $1.0 \times 10^{-2} \mathrm{M}$. 
Figure 1 shows the surface plasmon resonance spectra of freshly synthesized silver nanoparticles obtained with different reducing agent and concentration. The range of wavelength for spectra studied was 300-750 $\mathrm{nm}$. The surface plasmon resonance spectra of silver nanoparticles synthesized using $\mathrm{m}$ hydroxybenzoic are similar to those synthesized using p-hydroxybenzoic acid as reducing agent. The spectra display characteristic absorption spectra with absorbance maxima centered around 415-425 $\mathrm{nm}$. Absorption peak exhibit a slight red shift of its maximum from 420 to $424 \mathrm{~nm}$ and 415 to $421 \mathrm{~nm}$ by increasing the concentration of m-hydroxybenzoic acid and p-hydroxybenzoic acid respectively. The red-shift of $\lambda_{\max }$ has been associated with the increase in particle size (Krutyakov et al., 2008).

There is a small difference on $\square \max$ position of the obtained silver nanoparticles compared with those synthesized by others reducing agent. Nanoparticles synthesized using sodium borohydride as reducing agent and polyvinyl alcohol as capping agent exhibited the $\lambda_{\max }$ at $400 \mathrm{~nm}$ (Lee \& Meisel, 1982) and that of synthesized by reduction of silver ion with aniline and dodecylbenzene sulfonic acid as capping agent produced silver nanoparticles with surface plasmon resonance peak at $410 \mathrm{~nm}$ (Yang et al., 2011). The exact position of the $\lambda_{\max }$ is highly dependent on the size and shape of nanoparticles (Krutyakov et al., 2008; Mock et al., 2002). Silver nanoparticles with different size range can be generated if nanoparticles synthesized using different reducing agent (Agnihotri et al., 2014).

\section{Stability Study of Silver Nanoparticles}

The surface plasmon resonance spectra of silver nanoparticles were recorded during the time of the study. The changes of spectra including the position of $\lambda_{\max }$, intensity, and width of the peak were observed and presented in Figure 2. The results show that at the first 18 weeks period there are no significant changes in the spectra. It point that how surface plasmon resonance spectra of silver nanoparticles overlap which indicates the strong stability of silver nanoparticles against aggregation and dissolution. However, the slight differences of surface plasmon resonance spectra appear after 40 weeks of storage. For all silver nanoparticles, the position of $\lambda_{\max }$ is relatively constant with only changes in the range of 1-4 $\mathrm{nm}$. The highest shift of $\lambda_{\max }$ occurs in silver nanoparticles synthesized using $1 \times 10^{-3} \mathrm{M}$ m-hydroxybenzoic acid and $1 \times 10^{-2} \mathrm{M}$ p-hydroxybenzoic acid with $\lambda_{\max }$ shift from 420 to $424 \mathrm{~nm}$ and 421 to $417 \mathrm{~nm}$, respectively. The shift of $\lambda_{\max }$ indicates the change of particle size (Krutyakov et al., 2008).

The absorption peak intensity of silver nanoparticles surface plasmon resonance spectra underwent a small change after a period of 40 weeks. The intensity of silver nanoparticles synthesized using $1 \times 10^{-3} \mathrm{M} \mathrm{m}$ hydroxybenzoic acid and p-hydroxybenzoic acid decrease by $9.1 \%$ and $2.6 \%$. In contrast, the increase of peak intensity by $2.1 \%$ and $1.4 \%$ occur in silver nanoparticles synthesized using $1 \times 10^{-2} \mathrm{M}$ m-hydroxybenzoic acid and p-hydroxybenzoic acid. The decrease of peak intensity results from the change of nanoparticles size or dissolution of silver nanoparticles into a silver ion (Gunsolus, Mousavi, Hussein, Bühlmann, \& Haynes, 2015). In addition to the dissolution of particles, the formation of nanoparticles may occur during long-term storage (Pinto et al., 2010). The formation of nanoparticles occurs in the remaining silver ions present in colloidal nanoparticles and result in the increase of peak intensity.

In addition to the position of $\lambda_{\max }$ and absorption peak intensity, the FWHM of silver nanoparticles surface plasmon resonance spectra after 40 weeks also suffer a change compared with the fresh synthesized silver nanoparticles as shown in Figure 3. The FWHM of silver nanoparticles synthesized using $1 \times 10^{-2} \mathrm{M}$ p-hydroxybenzoic acid increase by $30 \%$ followed by silver nanoparticles synthesized using $1 \times 10^{-3} \mathrm{M}$ and $1 \times 10^{-2} \quad \mathrm{M}$ p-hydroxybenzoic $(24.0 \%$ and $22.1 \%$ ). An increase of only $6.7 \%$ occurs in silver nanoparticles synthesized using $1 \times 10^{-3} \mathrm{M}$ p-hydroxybenzoic acid. The increase of FWHM or broadening the surface plasmon resonance peak indicates the change in particle size distribution (Adegboyega et al., 2013). 
1

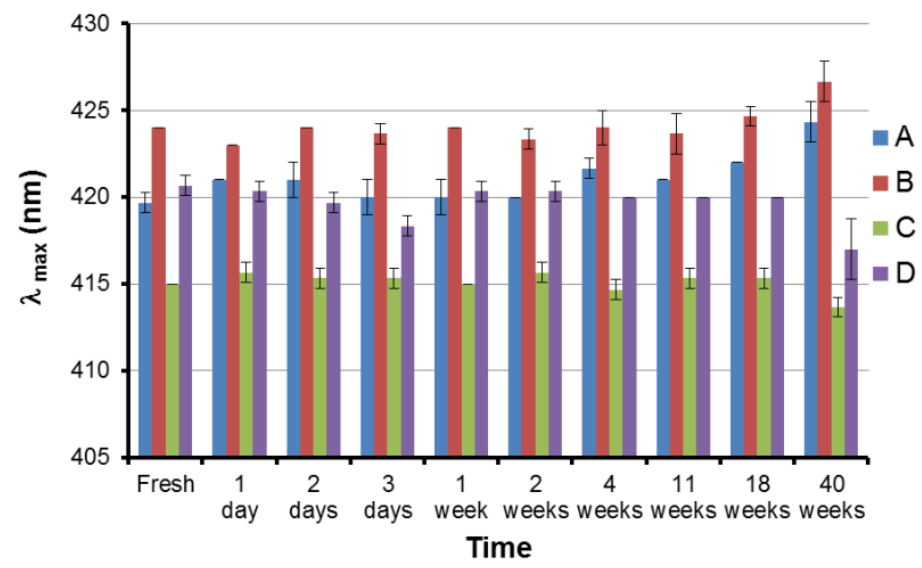

2

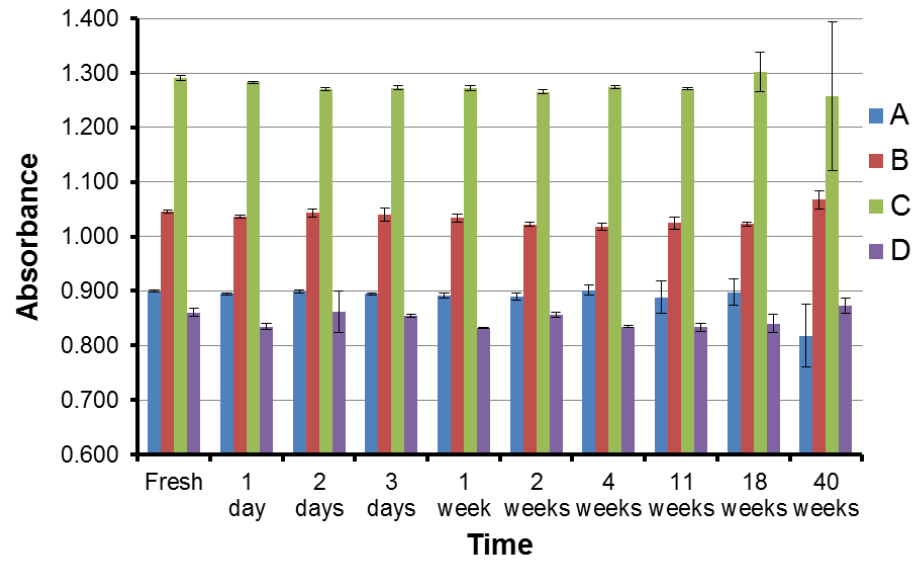

3

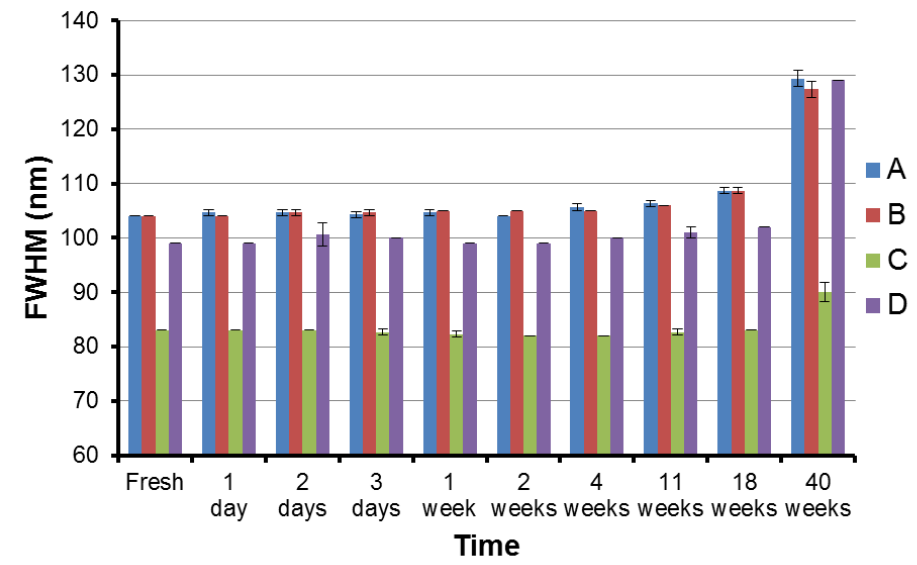

Figure 2. The changes of position of $\lambda_{\max }$ (1), peak intensity (2) ; and width of peak (3) of silver nanoparticles during the time of study. Silver nanoparticles were synthesized by reduction $2.5 \times$ $10^{-4} \mathrm{M}$ silver nitrate with A) m-hydroxybenzoic acid $\left.1.0 \times 10^{-3} \mathrm{M} ; \mathrm{B}\right)$ m-hydroxybenzoic acid $1.0 \times$ $10^{-2} \mathrm{M}$; C) p-hydroxybenzoic acid $1.0 \times 10^{-3} \mathrm{M}$; and D) p-hydroxybenzoic acid $1.0 \times 10^{-2} \mathrm{M}$. Replication $=3$

For silver nanoparticles suffering aggregation, there is change in particles size distribution which results in the distinct differences in surface plasmon resonance spectra. In addition to the broadening of absorption peak and reduction of primary absorption peak intensity around $400 \mathrm{~nm}$, the secondary absorption peak appears at the larger wavelength $(600 \mathrm{~nm})$. The secondary peak results from the scattering of light by larger particles. The primary absorption peak will disappear if total aggregation occurred (Gunsolus et al., 2015; Tejamaya et al., 2012).

Pinto et al. (2010) studied the stability of silver nanoparticles prepared by reduction silver ion with sodium borohydride in the presence of sodium citrate as a capping agent for a period of 1 year. After 1 year, silver 
nanoparticles suffered evolution in size and shape of particles in agreement with their surface plasmon resonance spectra with a secondary peak at larger wavelength was observed. The spectra also showed an increase of FWHM. Yang et al. (2011) synthesized silver nanoparticles by reduction of silver ion with aniline and dodecylbenzene sulfonic acid as capping agent. The silver nanoparticles were stable for more than 1 year at ambient temperature without any precipitation. Adegboyega et al. (2013) stated that silver nanoparticles prepared by reduction of silver ion with fulvic acids were highly stable over 180 days although a $15 \%$ peak intensity reduction was detected. Bastus et al. (2014) reported that silver nanoparticles prepared by reduction silver ion with the mixture of sodium citrate and tannic acid showed strong stability over a 7 weeks period.

Although there are little changes in the surface plasmon resonance spectra of silver nanoparticles synthesized by reduction of silver ion with m-hydroxybenzoic acid and p-

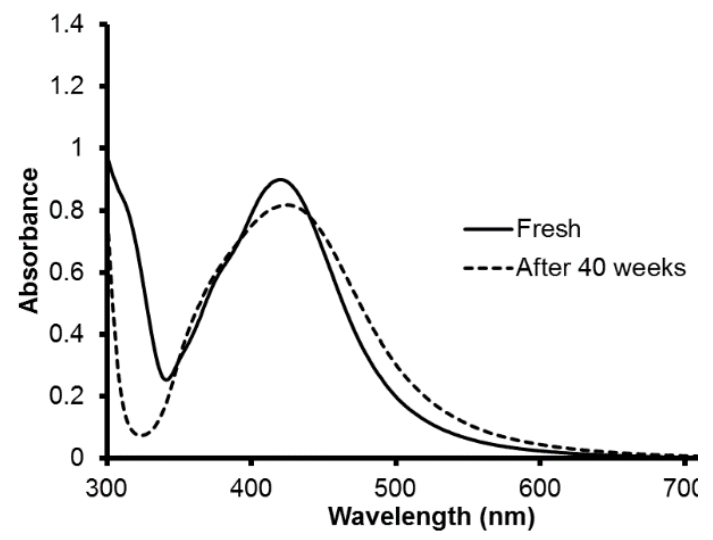

A

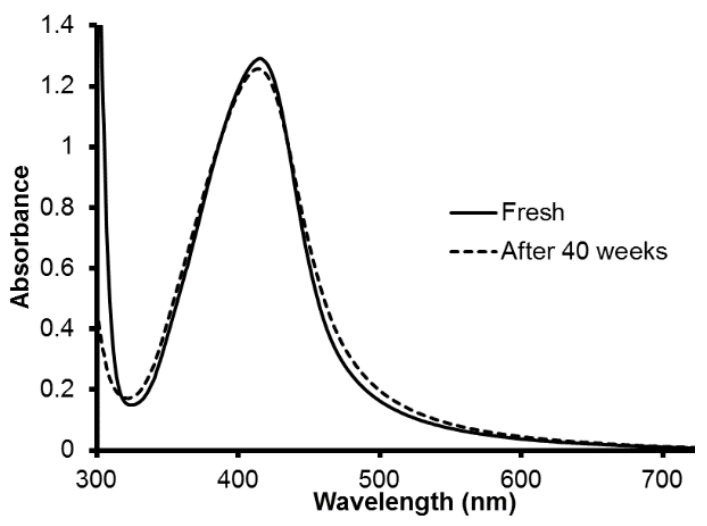

$\mathrm{C}$ hydroxybenzoic acid after storage for 40 weeks, it can be stated that silver nanoparticles capped with m-hydroxybenzoic acid and phydroxybenzoic acid show long-term high stability. It is supported by the fact that in addition to only $9.1 \%$ reduction of peak intensity, the primary peak in the region of $415-425 \mathrm{~nm}$ is still observed and there is no secondary peak appeared at the larger wavelength as shown in Figure 3. Since silver nanoparticles synthesized with no additional capping agent show the high stability over a long-term period of storage, it indicates that mhydroxybenzoic acid and p-hydroxybenzoic acid provide protecting effect for silver nanoparticles from aggregation, in addition, to act as a reducing agent. The FTIR spectra as shown in our previous published data (Gusrizal et al., 2016, 2017) indicated that mhydroxybenzoic acid and p-hydroxybenzoic acid were attached on the surface of silver nanoparticles through carboxylic group and prevent the aggregation of particles.

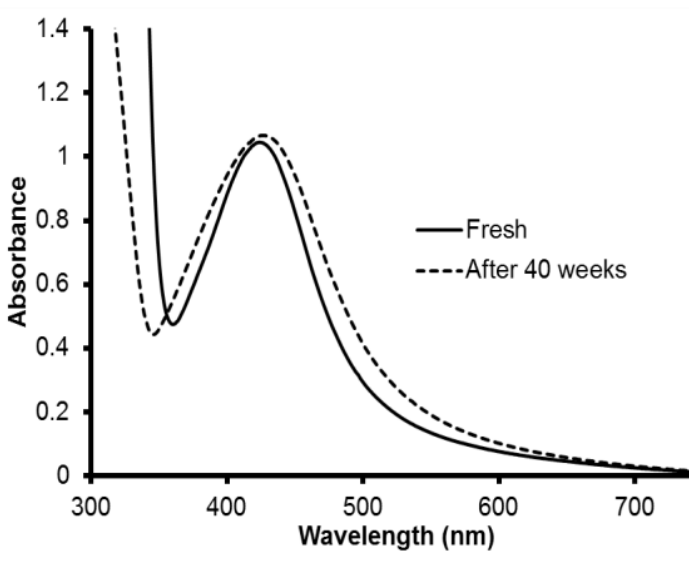

B

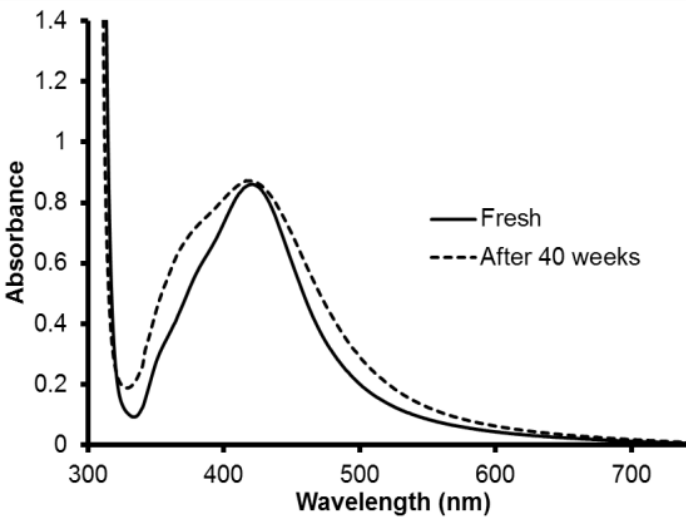

D

Figure 3. Surface plasmon resonance spectra of silver nanoparticles after 40 weeks storage. Silver nanoparticles were synthesized by reduction $2.5 \times 10^{-4} \mathrm{M}$ silver nitrate with A) m-hydroxybenzoic acid $1.0 \times 10^{-3} \mathrm{M}$; B) m-hydroxybenzoic acid $1.0 \times 10^{-2} \mathrm{M}$; C) p-hydroxybenzoic acid $1.0 \times 10^{-3} \mathrm{M}$; and D) p-hydroxybenzoic acid $1.0 \times 10^{-2} \mathrm{M}$ 


\section{CONCLUSIONS}

Two types of silver nanoparticles were successfully synthesized by reduction of silver nitrate with m-hydroxybenzoic acid and phydroxybenzoic acid without additional capping agent. Silver nanoparticles capped with m-hydroxybenzoic acid and phydroxybenzoic acid were proved to be highly stable for a period up to 40 weeks. It promises for them to be an exciting agent for further research and commercial application. The results show the potential of $\mathrm{m}$ hydroxybenzoic acid and p-hydroxybenzoic as a new reducing agent in the synthesis of highly stable silver nanoparticles. The $\mathrm{m}$ hydroxybenzoic acid and p-hydroxybenzoic acid act not only as reducing agent but also act simultaneously as capping agent in the synthesis of silver nanoparticles using chemical reduction approach.

\section{REFERENCES}

Adegboyega, N. F., Sharma, V. K., Siskova, K., Zbořil, R., Sohn, M., Schultz, B. J., \& Banerjee, S. (2013). Environmental Science and Technology, 47(2), 757764. https://doi.org/10.1021/es302305f

Agnihotri, S., Mukherji, S., \& Mukherji, S. (2014). Size-controlled silver nanoparticles synthesized over the range 5-100 nm using the same protocol and their antibacterial efficacy. RSC Advances, 4(8), 3974-3983. https://doi.org/10.1039/C3RA44507K

Annadhasan, M., Muthukumarasamyvel, T., Babu, V. R. S., \& Rajendiran, N. (2014). Green synthesized silver and gold nanoparticles for colorimetric detection of $\mathrm{Hg}^{2+}, \mathrm{Pb}^{2+}$, and $\mathrm{Mn}^{2+}$ in aqueous medium. ACS Sustainable Chemistry and Engineering, 2(4), 887-896. https://doi.org/10.1021/sc400500z

Bastus, N. G., Merkoçi, F., Piella, J., \& Puntes, V. (2014). Synthesis of highly monodisperse citrate-stabilized silver nanoparticles of up to $200 \mathrm{~nm}$ : Kinetic control and catalytic properties. Chemistry of Material, 26, 2836-2846. https://doi.org//10.1021/cm500316k

Chen, G., Roy, I., Yang, C., \& Prasad, P. N. (2016). Nanochemistry and nanomedicine for nanoparticle-based diagnostics and therapy. Chemical Reviews, 116(5), 2826-2885. https://doi.org/10.1021/acs.chemrev.5b0 0148

Gunsolus, I. L., Mousavi, M. P. S., Hussein, K., Bühlmann, P., \& Haynes, C. L. (2015). Effects of humic and fulvic acids on silver nanoparticle stability, dissolution, and toxicity. Environmental Science and Technology, 49(13), 80788086.

https://doi.org/10.1021/acs.est.5b01496

Gusrizal, G., Santosa, S. J., Kunarti, E. S., \& Rusdiarso, B. (2016). Dual function of p-hydroxybenzoic acid as reducing and capping agent in rapid and simple formation of stable silver nanoparticles. International Journal of ChemTech Research, 9(9), 472-482.

Gusrizal, G., Santosa, S. J., Kunarti E, S., \& Rusdiarso, B. (2017). Synthesis of silver nanoparticles by reduction of silver ion with m-hydroxybenzoic acid. Asian Journal of Chemistry, 29(7), 1417-1422. https://doi.org/10.14233/ajchem.2017.20 436

Krutyakov, Y. A., Kudrinskiy, A. A., Olenin, A. Y., \& Lisichkin, G. V. (2008). Synthesis and properties of silver nanoparticles: advances and prospects. Russian Chemical Reviews, 77(3), 233257.

https://doi.org/10.1070/RC2008v077n03 ABEH003751

Lee, P. C., \& Meisel, D. (1982). Adsorption and surface-enhanced Raman of dyes on silver and gold sols. Journal of Physical Chemistry, 86(17), 3391-3395. https://doi.org/10.1021/j100214a025

Marambio-Jones, C., \& Hoek, E. M. V. (2010). A review of the antibacterial effects of silver nanomaterials and potential implications for human health and the environment. Journal of Nanoparticles Research, 12, 1531-1551. https://doi.org/10.1007/s11051-0109900-y

Mock, J. J., Barbic, M., Smith, D. R., Schultz, D. A., \& Schultz, S. (2002). Shape effects in plasmon resonance of individual colloidal silver nanoparticles. Journal of Chemical Physics, 116(15), 6755-6759. https://doi.org/10.1063/1.1462610

Pinto, V. V., Ferreira, M. J., Silva, R., Santos, H. A., Silva, F., \& Pereira, C. M. (2010). Long time effect on the stability of silver 
nanoparticles in aqueous medium: Effect of the synthesis and storage conditions. Colloids and Surfaces A: Physicochemical and Engineering Aspects, 364(1-3), 19-25. https:// doi.org /10.1016/j.colsurfa.2010.04.015

Rycenga, M., Cobley, C. M., Zeng, J., Li, W., \& Moran, C. H. (2012). Controlling the synthesis and assembly of silver nanostructures for plasmonic applications. Chemical Reviews, 111(6), 3669-3712.

https://doi.org/10.1021/cr100275d

Sachdev, D., Kumar, V., Maheshwari, P. H., Pasricha, R., Deepthi, \& Baghel, N. (2016). Silver based nanomaterial, as a selective colorimetric sensor for visual detection of post harvest spoilage in onion. Sensors and Actuators, B: Chemical, 228(June), 471-479. https:// doi.org/10.1016/j.snb.2016.01.049

Shrivas, K., Sahu, S., Patra, G. K., Jaiswal, N. K., \& Shankar, R. (2016). Localized surface plasmon resonance of silver nanoparticles for sensitive colorimetric detection of chromium in surface water, industrial waste water and vegetable samples. Analytical Methods, 8(9), 2088-2096.

https://doi.org/10.1039/c5ay03120f

Sun, Y., \& Xia, Y. (2002). Shape-controlled synthesis of gold and silver nanoparticles. Science, 298(5601), 2176-2179.

https://doi.org/10.1126/science.1077229

Tejamaya, M., Römer, I., Merrifield, R. C., \& Lead, J. R. (2012). Stability of citrate, PVP, and PEG coated silver nanoparticles in ecotoxicology media. Environmental Science and Technology, 46(13), 7011-7017. https://doi.org /10.1021/es2038596

Wiley, B., Sun, Y., Chen, J., Cang, H., Li, Z. Y., Li, X., \& Xia, Y. (2005). Shapecontrolled synthesis of silver and gold nanostructures. MRS Bulletin, 30(5), 356-361. https://doi.org/10.1557/mrs2005.98

Yang, J., Yin, H., Jia, J., \& Wei, Y. (2011). Facile synthesis of high-concentration, stable aqueous dispersions of uniform silver nanoparticles using aniline as a reductant. Langmuir, 27(8), 5047-5053. https://doi.org/10.1021/la200013z 